\title{
Individualized Dosimetry of Kidney and Bone Marrow in Patients Undergoing ${ }^{177}$ Lu-DOTA-Octreotate Treatment
}

\author{
Mattias Sandström ${ }^{1,2}$, Ulrike Garske-Román ${ }^{2,3}$, Dan Granberg ${ }^{3}$, Silvia Johansson ${ }^{2}$, Charles Widström¹, Barbro Eriksson ${ }^{3}$, \\ Anders Sundin ${ }^{2,4}$, Hans Lundqvist ${ }^{5}$, and Mark Lubberink ${ }^{2}$ \\ ${ }^{1}$ Section of Medical Physics, Department of Radiology, Oncology, and Radiation Science, Uppsala University Hospital, Uppsala \\ University, Uppsala, Sweden; ${ }^{2}$ Section of Nuclear Medicine and PET, Department of Radiology, Oncology, and Radiation Science, \\ Uppsala University Hospital, Uppsala University, Uppsala, Sweden; ${ }^{3}$ Section of Endocrine Oncology, Department of Medical \\ Sciences, Uppsala University Hospital, Uppsala University, Uppsala, Sweden; ${ }^{4}$ Department of Radiology, Molecular Medicine, and \\ Surgery, Karolinska Institute, Stockholm, Sweden; and ${ }^{5}$ Rudbeck Laboratory, Section of Biomedical Radiation Sciences, Department \\ of Radiology, Oncology, and Radiation Science, Uppsala University, Uppsala, Sweden
}

The organs at risk in radionuclide therapy with ${ }^{177}$ Lu-octreotate are the bone marrow and the kidneys. The primary aim of this study was to develop an individualized dosimetry protocol for the bone marrow. The secondary aim was to identify those patients, undergoing fractionated therapy with $7.4 \mathrm{GBq} / \mathrm{cycle}$, who first reached an accumulated dose of either 2 Gy to the bone marrow or 23 Gy to the kidneys. Methods: Two hundred patients with metastatic neuroendocrine tumors with high somatostatin receptor expression were included. After the administration of $7.4 \mathrm{GBq}$ of ${ }^{177} \mathrm{Lu}$-octreotate, blood samples were drawn 6 times within the first 24 h. In 50 patients, additional blood samples were obtained at 96 and $168 \mathrm{~h}$. Moreover, urine was collected from 30 patients during the first $24 \mathrm{~h}$. Planar whole-body and SPECT/CT images over the abdomen were acquired at 24, 96, and $168 \mathrm{~h}$ after the infusion. Calculation of the absorbed radiation dose to the bone marrow was based on blood and urinary activity curves combined with organ-based analysis of the whole-body images. The absorbed dose to the kidney was calculated from the pharmacokinetic data obtained from SPECT/CT. Results: For a single cycle of $7.4 \mathrm{GBq}$, the absorbed dose to the bone marrow and the kidney ranged from 0.05 to $0.4 \mathrm{~Gy}$ and from 2 to $10 \mathrm{~Gy}$, respectively. In 197 of 200 patients, the kidneys accumulated an absorbed dose of 23 Gy before the bone marrow reached 2 Gy. Between 2 and 10 cycles of ${ }^{177} \mathrm{Lu}$-octreotate could be administered before the upper dose limit for the individual patient was reached. Conclusion: A method based on repeated whole-body imaging in combination with blood and urinary activity data over time was developed to determine the absorbed dose to the bone marrow. The dose-limiting organ was the kidney in 197 of 200 patients. In $50 \%$ of the patients, more than 4 cycles of 7.4 GBq of ${ }^{177}$ Lu-octreotate could be administered, whereas $20 \%$ of the subjects were treated with fewer than 4 cycles. Individualized absorbed dose calculation is essential to optimize the therapy.

Received Apr. 17, 2012; revision accepted Aug. 21, 2012.

For correspondence or reprints contact: Mattias Sandström, Uppsala University Hospital, Section of Medical Physics, SE-751 85 Uppsala, Sweden.

E-mail: mattias.sandstrom@akademiska.se

Published online Dec. 7, 2012.

COPYRIGHT @ 2013 by the Society of Nuclear Medicine and Molecular Imaging, Inc.
Key Words: individualized; dosimetry; ${ }^{177}$ Lu-DOTA-octreotate; bone marrow; kidney

J Nucl Med 2013; 54:33-41

DOI: 10.2967/jnumed.112.107524

$\mathbf{T}$ reatment failure in cancer is often attributed to insufficient control of metastatic disease. To address this problem, new therapeutic modalities are being developed, among them targeted radionuclide therapy. Small peptides binding to somatostatin receptors (octreotide and octreotate) can be labeled with radioactive metal ions and used for both receptor imaging and radiation therapy. Peptide receptor radionuclide therapy (PRRT) targeting this receptor group for treatment of neuroendocrine tumors has shown promising results (1-5).

The best results in targeted radionuclide therapy are obtained when the absorbed dose is close to, but not exceeding, the maximum acceptable for radiosensitive organs. If absorbed doses to risk organs are unknown, a considerable safety margin in the administered activity amount is warranted. Consequently, all too often this results in an undesirably low absorbed dose to tumor and the risk for suboptimal treatment (6). In external radiation therapy, the absorbed dose to organs at risk varies with their proximity to the radiation field. In PRRT, the individual kinetics of the used peptide determines the tissue activity distribution. Hence, the absorbed dose to organs and tumors is governed by a complex combination of factors that at present is difficult to predict. The main organs at risk are usually kidneys and bone marrow $(5-10)$.

In PRRT with ${ }^{177} \mathrm{Lu}$-octreotate, most published data are based on a fixed treatment schedule using 4 consecutive cycles of $7.4 \mathrm{GBq}$, because this amount of accumulated activity has been shown to be safe for most patients $(1,2)$. Personalized dosimetry, to identify dose-limiting organs and maximum tolerated accumulated activity for the individual patient, would therefore most likely be an improvement. 
Earlier experience from external-beam radiation therapy and radioiodine treatment of the thyroid is today used as a guide to establish the maximum absorbed dose limits to the kidneys and bone marrow. However, the treatment situation for the individual patient is subjected to dynamic changes in tumor size, organ function, and, in the case of neuroendocrine tumors, hormone levels. All these factors have an impact on the absorbed doses during consecutive treatment cycles. The biologic relation between the absorbed dose and the clinical effect is also dependent on other, partly unknown, factors such as the dose rate, the intracellular distribution of the radionuclide, and the tumor's radiosensitivity (10-12). Consequently, the concept of performing individual dosimetry is an important step toward gaining knowledge on how to optimize PRRT.

To accurately calculate absorbed dose to organs at risk (bone marrow, kidneys, spleen, and liver), the kinetics of the administered radionuclide need to be known (13). Guidelines on how dosimetry should be performed in PRRT are found in the literature $(13,14)$. When radionuclides emitting both charged particles and photons are used for treatment, dosimetry for solid organs with a high activity concentration is different from that for radiation-sensitive organs with low uptake. ${ }^{177} \mathrm{Lu}$ decays solely by $\beta$-decay and emits low-energy charged particles ( $\beta$-radiation, Auger electrons, and conversion electrons) with a mean energy of $148 \mathrm{keV}$ per decay and endpoint energy of the main $\beta$-radiation of $498 \mathrm{keV}$ (abundance $78.6 \%$ ). The energies of the main $\gamma$-radiation are 208 and $113 \mathrm{keV}$, emitted in $11.0 \%$ and $6.4 \%$ of decays, respectively. In tissues with high uptake such as kidney, liver, spleen, and tumor, the cross-fire from surrounding low-uptake areas can be neglected because of the local absorption of $\beta$-radiation, combined with the comparably low absorption of $\gamma$-radiation (15). Correspondingly, in smaller organs only a fraction $(<2 \%)$ of the photon energy is absorbed locally. On the other hand, areas of high activity concentration contribute substantially to the absorbed dose in organs with low activity concentration such as the bone marrow.

Forrer et al. (16) showed that the activity concentration measured in bone marrow aspirates between days 4 and 8 of the treatment was equal to that in blood. Thus, self-dose to bone marrow can simply be calculated by integrating the blood kinetics. To calculate the absorbed dose from cross radiation, the total activity over time from adjacent organs as well as from the remainder of the body has to be known. To minimize possible bone marrow complications, a limit of the absorbed dose to the bone marrow of $2 \mathrm{~Gy}$ has been suggested (17).

Several studies have reported dosimetry data for the kidney based on 2-dimensional (2D) $(1,8,16,18)$ or 3-dimensional (3D) (19-22) imaging and attempted to tackle the question of the dose-limiting organ. In conventional external-beam radiotherapy, an absorbed dose limit of $23 \mathrm{~Gy}$ is used for the kidney (23). Because of the lower dose rates in PRRT, higher absorbed doses can probably be accepted, and Konijnenberg et al. (24), in view of the results of 3D imaging, argued for a limit of 29 Gy to the kidneys. In the present study, a cautious approach was adopted by accepting $23 \mathrm{~Gy}$ as the limit for the absorbed dose. To create feasible dosimetry for solid organs, we have previously proposed a protocol based on complete dosimetry during the first therapy cycle combined with single SPECT/CT scans at $24 \mathrm{~h}$ after injection in subsequent cycles (25). In these subsequent cycles, absorbed doses to kidneys, liver, and spleen are then calculated under the assumption of unchanged kinetics compared with the first cycle (25).

The main aim of this paper was to develop an individualized and pragmatic dosimetry of the bone marrow in patients with metastatic neuroendocrine tumors who were repeatedly treated with ${ }^{177} \mathrm{Lu}$-DOTA-Tyr-3-octreotate. The change of the absorbed dose to the bone marrow during treatment was also assessed. The secondary aim was to determine the doselimiting organ for fractionated therapy with $7.4 \mathrm{GBq} / \mathrm{cycle}$ and the maximal number of cycles in the individual patient, accepting an accumulated dose of 23 Gy to the kidneys and 2 Gy to the bone marrow. To accomplish this aim, the results of the bone marrow dosimetry were combined with those of the $3 \mathrm{D}$ dosimetry protocol for the kidney as described earlier (20).

\section{MATERIALS AND METHODS}

\section{Patients and Therapy}

Two hundred patients (98 women and 102 men) with metastatic somatostatin receptor-expressing neuroendocrine tumors treated with ${ }^{177} \mathrm{Lu}$-DOTA-Tyr-3-octreotate were included. All patients met the inclusion criteria described earlier (20). Since September 2010, all patients had been included into a prospective study (EudraCT no. 2009-012260-14) approved by the Regional Ethical Review Board in Uppsala after giving written informed consent. Before that time, patients were admitted on a single-patient basis for compassionate use with individual permission of the Swedish Medical Products Agency. DOTA-Tyr-3-octreotate was a generous gift from Prof. Eric Krenning (Erasmus Medical Centre). ${ }^{177} \mathrm{LuCl}_{3}$ was purchased from IDB. Labeling was performed in-house before the infusion. Quality control was performed on each lot using thin-layer chromatography, and only labeling yields over $97 \%$ were accepted for treatment. On a regular basis, a high-performance liquid chromatography quality control test was performed. The radioactive ligand, diluted in $100 \mathrm{~mL}$ of saline, was infused intravenously during $30 \mathrm{~min}$ followed by a 15 -min rinsing of the tubing with physiologic saline. An intravenous infusion of a mixed amino acid solution (Vamin $14 \mathrm{~g} \mathrm{~N} / \mathrm{L}$ electrolyte-free; Fresenius Kabi) was started half an hour before the activity administration at a rate of $250 \mathrm{~mL} / \mathrm{h}$. One hundred thirtyfour of 200 patients received $1 \mathrm{~L}$; the later patients received $2 \mathrm{~L}$ of amino acid solution as standard protocol.

\section{Image Acquisition}

All patients underwent whole-body $\gamma$-scintigraphy (anterior and posterior planar acquisitions) and SPECT/CT of the abdomen at 24, 96, and $168 \mathrm{~h}$ after administration of the first therapeutic dose of $7.4 \mathrm{GBq}$ of ${ }^{177} \mathrm{Lu}$-DOTA-Tyr-3-octreotate. For the first 69 patients, imaging was performed on a Hawkeye Millennium VG (GE Healthcare) dual-head $\gamma$-camera equipped with $1.6-\mathrm{cm}(5 / 8$-in) $\mathrm{NaI}(\mathrm{Tl})$ crystals and VPC-5 (medium-energy general-purpose) collimators. A $20 \%$ energy window around the 2 dominant $\gamma$-ray energies of ${ }^{177} \mathrm{Lu}, 113.0$ and $208.4 \mathrm{keV}$, was applied. For the other 131 patients, imaging was performed on an Infinia (GE Healthcare) 
dual-head $\gamma$-camera equipped with $1.0 \mathrm{~cm}(3 / 8$-in) $\mathrm{NaI}(\mathrm{Tl})$ crystals and VPC-5 (medium-energy general-purpose) collimators. A $20 \%$ energy window was placed around the dominant 208.4-keV $\gamma$-ray energy of ${ }^{177} \mathrm{Lu}$ to make the measurements. Imaging with wholebody scintigraphy and SPECT/CT was performed as described earlier (20) with the exception that SPECT/CT images were collected with 120 angles and $30 \mathrm{~s}$ per frame for the Infinia. Calibration of wholebody and SPECT images was based on a $100-\mathrm{mL}$ sphere containing a known amount of activity placed inside a thorax phantom, which was scanned repeatedly (20). Phantom measurements confirmed that there were no dead-time issues in the patient measurements.

\section{Blood Measurements}

Blood samples $(\sim 3 \mathrm{~g})$ were drawn at $0.5,1.0,2.5,4,8$, and $24 \mathrm{~h}$ after the start of ${ }^{177}$ Lu-DOTA-Tyr-3-octreotate administration. In the first 50 patients, additional samples were collected at 96 and $168 \mathrm{~h}$ after injection. Samples were weighed with a precision of $1 \mathrm{mg}$ (PJ 300; Mettler Toledo). For the first 121 patients, a reference sample of about $5 \mathrm{mg}$ of the infused activity solution was weighed with a precision of $0.01 \mathrm{mg}$ (AE 163; Mettler Toledo) and measured together with blood samples in an automatic well counter (Wizard 3"; Perkin Elmer) to determine the absolute activity concentrations in the blood samples. For the other patients, the automatic well counter was absolutely calibrated for ${ }^{177} \mathrm{Lu}$, and activity concentrations in the samples were obtained directly.

The blood activity concentration was integrated using the trapezoidal method. In $5 \%$ of the patients, the blood kinetics could not be described by a biexponential function, which is why trapezoidal integration was used to estimate the time-integrated activity concentration for the first $24 \mathrm{~h}$. Of the patients for whom late blood samples were taken, $95 \%$ showed a half-life in blood for the second phase (after $24 \mathrm{~h}$ ) shorter than $72 \mathrm{~h}$. The late blood phase in all patients was hence assumed to follow a single exponential curve and was integrated between $24 \mathrm{~h}$ and infinity using an effective half-life of $72 \mathrm{~h}$. Assuming that the activity concentration in bone marrow was the same as in blood (16), the time-integrated activity concentration obtained for blood was also applied for calculation of the bone marrow self-dose.

\section{Urine Samples}

In 30 patients, for practical reasons from male patients only, urine was collected from the start of infusion and during approximately $24 \mathrm{~h}$. The first 8 urinations were collected in separate vessels while further urinations were pooled in a ninth container. All containers were weighed. Samples of about $0.1 \mathrm{~g}$, weighed with a precision of $1 \mathrm{mg}$ (PJ 300), were taken from each container and subsequently diluted to a total volume of $3 \mathrm{~mL}$ before being measured in an automatic well counter (Wizard 3", Perkin Elmer). From these samples, the total amount of excreted activity from the body during the first $24 \mathrm{~h}$ was calculated and added to the measured total body activity from whole-body scintigraphy after $24 \mathrm{~h}$. The value obtained was compared with the administered activity to ensure that the 2 measurements gave an activity recovery close to $100 \%$.

\section{Image Analysis}

Further analysis of the images was performed using software developed in-house within the Hermes platform on an HNAC workstation with Gold 2.9 (Hermes). For dosimetry of solid organs, spheric volumes of interest $\left(4 \mathrm{~cm}^{3}\right)$ were placed on attenuationcorrected SPECT images over healthy kidney, liver, and spleen tissue, avoiding tumor tissue. Activity concentrations were determined for each time point, and time-integrated activity concentration was calculated as the area under the curve of a single exponential fit to the time-activity concentration curve.

For bone marrow dosimetry, regions of interest were drawn on geometric mean images obtained by planar whole-body scans at $24 \mathrm{~h}$ to delineate kidney, liver, spleen, tumor, and whole body. These regions of interest were subsequently transferred to the 96- and 168$\mathrm{h}$ images, and the data from the kidney, liver, spleen, and tumor were fitted to a monoexponential function.

The activity clearance from the remainder of the body was calculated on the basis of urine measurements during the first $24 \mathrm{~h}$ and whole-body scintigraphy for the later times. For the 30 patients from whom urine was collected, the activity in the total body during the first $24 \mathrm{~h}$ was calculated as the administered activity minus the accumulated urine activity, resulting in a total body time-activity curve for the first $24 \mathrm{~h}$. Kidney, liver, spleen, and tumor activities were subtracted using the monoexponential fits to the time-activity curves of these organs. From the graph obtained, a conservative half-life of $2 \mathrm{~h}$ for the early phase (0-24 h) was estimated and then used for all patients from whom urine was not collected, whereas the second phase was individually determined from the whole-body scintigraphy measurements at 24,96 , and $168 \mathrm{~h}$ minus the kidney, liver, spleen, and tumor activities.

\section{Absorbed Dose Calculations}

Absorbed doses to the kidneys, liver, and spleen were calculated by multiplication of organ time-integrated activity concentration by the appropriate dose concentration factor (DCF), considering only self-dose.

$$
\mathrm{D}_{\mathrm{O}}=\mathrm{DCF} \times \mathrm{C}_{\text {cumO }}, \quad \text { Eq. } 1
$$

where $D_{O}$ is the absorbed dose to the organ, $C_{\text {cumo }}$ is the timeintegrated activity concentration in the organ, and dose concentration factor is the factor converting $\mathrm{C}_{\mathrm{cumO}}$ to absorbed dose by selfabsorption.

For calculation of the absorbed dose to bone marrow, the contribution of other source organs was added to the self-dose derived from the blood measurements as follows:

$$
\mathrm{D}_{\mathrm{BM}}=\mathrm{DCF} \times \mathrm{C}_{\text {cumBM }}+\sum \mathrm{DF}_{\mathrm{BM} \leftarrow \mathrm{T}} \times \mathrm{A}_{\text {cumT }}, \quad \text { Eq. } 2
$$

where $\mathrm{D}_{\mathrm{BM}}$ is the absorbed dose to the bone marrow, $\mathrm{C}_{\text {cumBM }}$ is the time-integrated activity concentration in the bone marrow, dose concentration factor is the factor converting $\mathrm{C}_{\text {cumBM }}$ to absorbed dose by self absorption, $\mathrm{A}_{\text {cumT }}$ is the time-integrated activity in tissue $\mathrm{T}$ (solid organs, tumor, and remainder of the body), and $\mathrm{DF}_{\mathrm{BM} \leftarrow \mathrm{T}}$ is the factor converting $\mathrm{A}_{\text {cumT }}$ to absorbed dose in the bone marrow (cross-fire). Because most patients had significant tumor uptake, tumor was included as an additional virtual source organ. Since most of the tumors were found close to the liver and spleen, the mean DF of these organs was used to describe cross-dose from tumors to bone marrow. As stated by Stabin (26), MIRDOSE3 and its successor OLINDA/EXM 1.0 overestimate cross-doses from the remainder of the body to bone marrow since its DFs include a $\beta$-component, which should be subtracted if there is no uptake of ${ }^{177} \mathrm{Lu}$ in bone. Therefore, we applied cross-radiation absorbed fractions based on the $\gamma$-fraction of the DFs in OLINDA/EXM. The DFs were also confirmed by calculations using the $\gamma$-DFs given on the RADAR Web site (27).

For the 200 patients, the individual absorbed doses for the kidneys, the liver, and the spleen were plotted and the means, medians, and 
first and third quartiles were calculated. Moreover, the individual absorbed doses of bone marrow and how different tissues contributed were plotted.

\section{Changes in Absorbed Dose to Bone Marrow at Later Treatment Cycles}

Treatment changed the organ and tumor uptake as well as the elimination of the administered activity in consecutive cycles, for some patients in a dramatic way. This change also influenced the absorbed dose in the consecutive therapy cycles, as previously reported for the dosimetry of the solid organs in 30 patients (25). For the same patients a similar comparison of the bone marrow dose was performed. Data on absorbed doses obtained at therapy cycle 4 or later were divided by those of the first cycle, resulting in the ratio $R_{\text {dose }}=\frac{D_{4}}{D_{1}}$. The individual data from the 30 patients were plotted, and the means, medians, and first and third quartiles of the ratios were calculated.

\section{Dose-Limiting Organ}

The maximum number of therapy cycles to each individual patient was determined by the organ that first obtained its accepted tolerance dose, either 23 Gy to the kidneys or 2 Gy to the bone marrow. Since 3D dosimetry data were not available for the kidney at each therapy cycle, a dose approximation for later cycles was performed whereby an increase of the absorbed dose of 5\% was assumed for each consecutive therapy cycle. This estimate was based on our earlier published results showing a mean increase of $20 \%$ for the absorbed dose to the kidney between cycles 1 and 4 (25). For the bone marrow, no such correction was performed. The fraction of patients was calculated in which the kidney and bone marrow, respectively, was the dose-limiting organ.

\section{Statistical Methods}

Data were analyzed with an Anderson-Darling test to check for normal distribution. Since merely about half of the datasets passed the test, all data were analyzed as being nonnormally distributed. All imaging data were fitted to exponential functions using the least-square method. The coefficient of multiple determination $\left(R^{2}\right)$ was calculated for all imaging data. A nonparametric Wilcoxon paired test was applied to test, first, differences between the measured total body activity at $24 \mathrm{~h}$ after injection and injected activity minus all activity collected in urine during $24 \mathrm{~h}$, second, the absorbed dose to the bone marrow at a later cycle compared with that of the first cycle, and, third, the data for the dose-limiting organ. The median, first quartile, and the third quartile were calculated for the 2 phases of the effective half-life of the fit to the biexponential function of the blood concentration and the remainder of the body. Where applicable, data are presented as median (first to third quartiles). Statistical significance was assumed at a $P$ value of less than 0.05 .

\section{RESULTS}

\section{Kinetics}

Figure 1A shows a typical time-activity curve of the blood activity concentration and the biexponential function fitted to the data (patient 5). The effective half-lives were $1.61 \mathrm{~h}$ (range, $1.44-1.83 \mathrm{~h}$ ) for the first phase and $42.9 \mathrm{~h}$ (range, 38.8-51.4 h) for the second phase. An example of the kinetics of the activity excretion from the remainder of the body derived from urine measurements (0-24 h) and whole-body measurements $(24,96$, and $168 \mathrm{~h})$ is shown in Figure 1B (patient 159). These data are described by a biexponential function, with effective half-lives of $1.28 \mathrm{~h}$ (range, $0.93-1.52 \mathrm{~h}$ ) and $49.5 \mathrm{~h}$ (range, $45.1-56.6 \mathrm{~h}$ ) for the 2 elimination phases.

The early activity clearance in the remainder of the body (derived from the urine samples) and blood were similar, with the half-life ratio (calculated in 30 patients) having a median of 1.09 (range, 0.76-1.32).

For the measured data, fitted to a biexponential function (blood and remainder of the body) and a single-exponential function (small volumes of interest in the SPECT images and regions of interest in the geometric mean images), the coefficient of multiple determination $\left(R^{2}\right)$ was calculated to check the correspondence between the fitted curve and the measured data and was generally greater than 0.99 .

The activity excreted into the urine during the first $24 \mathrm{~h}$ added to the 24-h total-body activity as measured with the planar $\gamma$-camera was found to add up to 7.4 GBq (range, 7.27.6 GBq), which corresponded to the administered activity of 7.4 GBq. However, there was a large variation between patients regarding the excretion rate, as illustrated in Figure 2. There was a strong correlation between the 2 methods used to calculate the whole-body activity $\left(R^{2}=0.90\right.$ and slope of 1.00).

\section{Absorbed Doses}

The absorbed dose to bone marrow from the first therapy cycle is shown in Figure 3 and was less than 0.2 Gy (median, 0.12; interquartile range, 0.09-0.16) in most patients $(176 / 200)$. Figure 4 shows the contributions to the absorbed dose in the bone marrow from different tissues. In most (167/200) patients, the largest contribution was derived from the locally absorbed particles (self-dose) conveyed by the blood, followed by cross-doses from the remainder of the
FIGURE 1. Typical blood clearance curve in patient treated with ${ }^{177} \mathrm{Lu}$-octreotate $(\mathrm{A})$ and typical clearance curve of activity in total body (B).
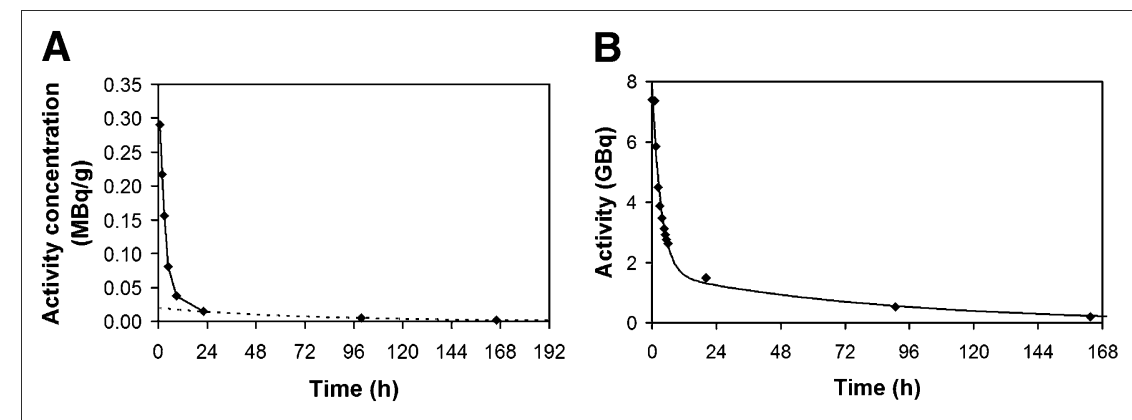


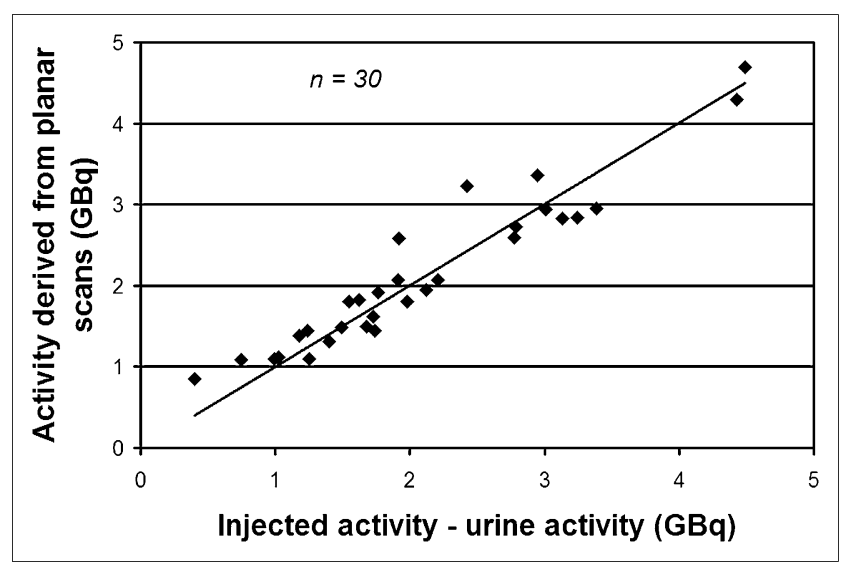

FIGURE 2. Correlation of activity obtained in whole-body imaging vs. activity obtained by subtracting urine from administered activity measured in 30 patients.

body and the tumors. The high contribution of the liver in some patients could be explained by a high tumor burden in the liver, which was difficult to differentiate from tumorfree liver tissue. Absorbed doses per cycle to kidneys, liver, and spleen are shown in Figure 5 and Table 1.

\section{Changes in Absorbed Dose of Bone Marrow at Later Treatment Cycles}

The ratio between the absorbed doses to the bone marrow at later therapy cycles divided by that of the first cycle is shown in Figure 6. The median ratio was 0.82 (interquartile range, 0.64-0.98), and the reduction in absorbed dose was significant $(P<0.001)$. The interquartile range of this ratio (difference between the first and third quartiles) was 0.34 for the bone marrow and 0.39 for both kidneys.

\section{Dose-Limiting Organ}

When accepting a maximum tolerated absorbed dose of 2 Gy to the bone marrow or 23 Gy to the kidneys, the doselimiting organ was the kidney in $98.5 \%$ of the patients and the bone marrow in only $1.5 \%(P<0.001)$. Even when a higher maximum kidney dose of 29 Gy was applied, the

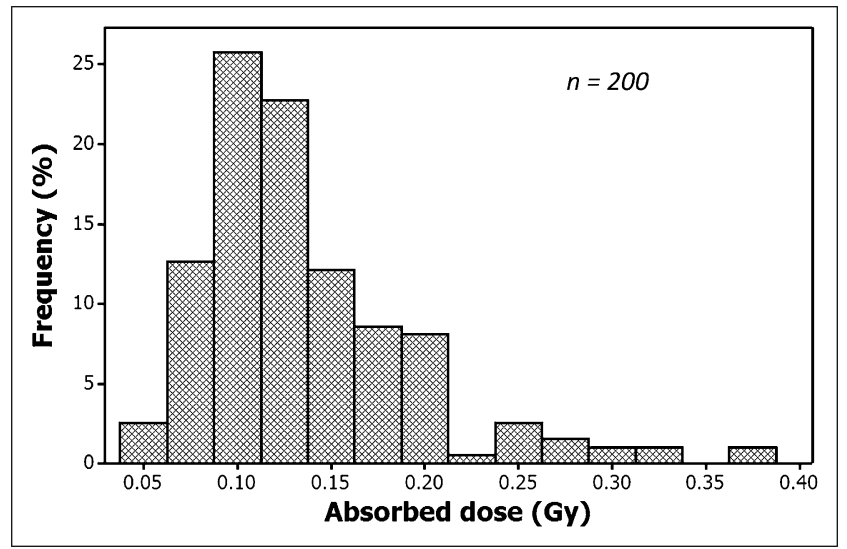

FIGURE 3. Total absorbed dose to bone marrow of 200 patients for a single therapy cycle. kidneys remained dose-limiting in $98 \%$ of the patients. The maximum number of tolerable therapy cycles of $7.4 \mathrm{GBq}$ per patient with the 2- and 23-Gy limits ranged from 2 to 10 cycles (median, 4.53; interquartile range, 3.87-5.52) (Fig. 7). According to this result, $50 \%$ of the patients could receive more than 4 cycles.

\section{DISCUSSION}

Here we have presented the largest, to our knowledge, cohort of patients with neuroendocrine tumors undergoing therapy with ${ }^{177} \mathrm{Lu}$-DOTA-octreotate and applying individual dosimetry. We have described a method for bone marrow dosimetry based on blood sampling and whole-body imaging complemented by urine sampling in a subset of patients.

Large clinical series on ${ }^{177} \mathrm{Lu}$-DOTA-octreotate therapy have shown favorable clinical outcome but have not reported data on individualized dosimetry $(2,5)$. Experts in the field have gradually come to an agreement that dosimetry is inevitable in order to optimize PRRT, and there is an ongoing discussion on how dosimetry should be performed and which is the most adequate model to apply $(12,28-31)$. An important point in this discussion is how to implement dosimetry best in daily clinical practice. A delicate balance between treatment efficacy, patient safety, and clinical applicability needs to be maintained.

In radionuclide therapy with small peptides such as ${ }^{177} \mathrm{Lu}$-DOTA-octreotate, the dose-limiting organs are in most patients the kidneys or the bone marrow $(2,4,5,7,8,10,16)$. Earlier published work on kidney toxicity outlined the obstacles of kidney dosimetry and radiation protection $(9,11,32-34)$. Coadministration of amino acids has decreased the doses to the kidney parenchyma effectively and improved the toxicity profile of the treatment. Almost half (43\%) of 310 patients treated with ${ }^{177} \mathrm{Lu}$-DOTA-octreotate by the group in Rotterdam (2), and all of the 1,109 patients treated with ${ }^{90}$ Y-DOTATOC by the group in Basel (5), were in clinical

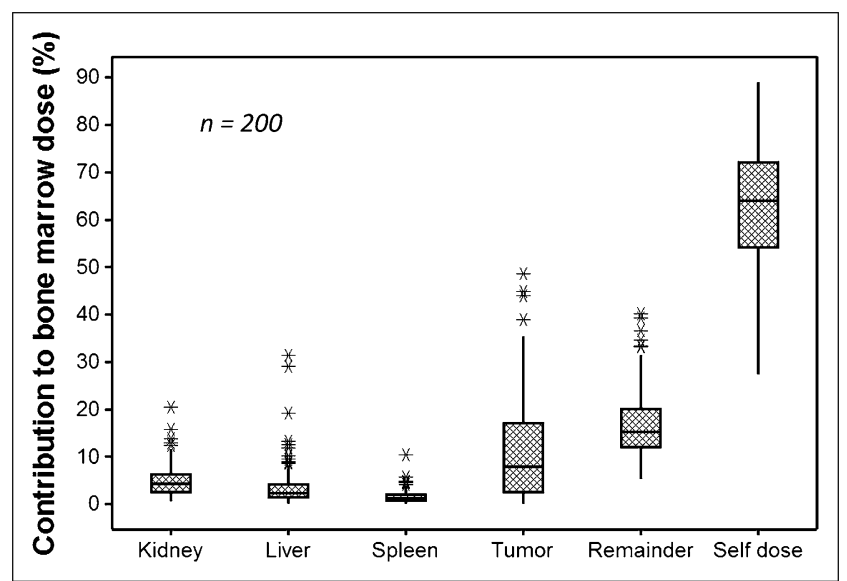

FIGURE 4. Relative contribution to bone marrow dose from different sources for 200 patients. Boxes indicate first and third quartiles, whisker lengths correspond to maximum and minimum data points with \pm 1.5 times height of box from first and third quartiles, and asterisks indicate values outside this range. 


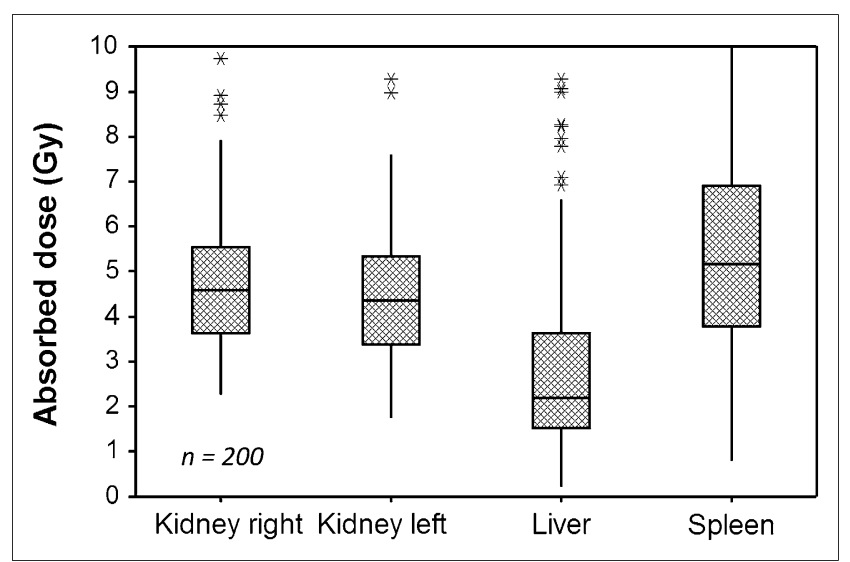

FIGURE 5. Absorbed doses to solid organs of 200 patients for a single therapy cycle. Boxes indicate first and third quartiles, whisker lengths correspond to maximum and minimum data points with \pm 1.5 times height of box from first and third quartiles, and asterisks indicate values outside this range.

progression at the start of therapy. The collective experience in major reports on PRRT and in our own report is that there are many patients with disseminated neuroendocrine tumors with virtually no other treatment options that respond to PRRT. Additionally, there is increasing evidence that patients during treatment with ${ }^{177} \mathrm{Lu}$-DOTA-octreotate generally experience an improved quality of life (35).

Despite these promising results, ultimately, most patients still die from lack of control of their disease. In these clinical trials on PRRT, little toxicity from the treatment was reported. Together with the fact that patients generally receive a standard treatment that comprises fixed amounts of ${ }^{177} \mathrm{Lu}$-DOTAoctreotate or ${ }^{90}$ Y-DOTATOC, this lack of toxicity indicates that there is room to optimize the method in terms of maximizing the tumor dose. The goal for research in this field is therefore to create a valid, yet practical, method to estimate the tolerance of the individual patient to the radiation delivered. Our approach was to establish a clinically feasible dosimetry method that measures the changes in activity concentration and absorbed dose in organs at risk in patients undergoing PRRT with ${ }^{177} \mathrm{Lu}$-octreotate. A dosimetry model for solid organs and tumors based on repeated 3D imaging was proposed earlier by our group (20). In this paper, the goal was to introduce a model for bone marrow dosimetry that would work equally well.

In clinical practice, dosimetry has most often been performed on the basis of $2 \mathrm{D}$ imaging to measure the activity in solid organs at several time points after administration of therapy $(1,18,36)$ In the present patient group, this approach would have 2 major drawbacks. First, organs and tumor overlap in many patients, and organs often contain tumor tissue, preventing exact uptake measurements (19-21). Furthermore, the organ sizes may vary substantially (20), and from 2D images it is not possible to estimate organ thicknesses or volumes. For this reason, the absorbed dose in solid organs was in the present work based on activity concentrations measured in attenuation-corrected SPECT images, and 2D imaging was used only to calculate activity in the remainder of the body and total organ activity to assess cross-dose to bone marrow. The absorbed dose to the kidneys was obtained by placing small spheres in apparently normal kidney tissue at 3 time points during the treatment cycle. The derived activity concentration as a function of time was integrated to obtain the total number of decays per gram of tissue. Because the cross-fire contribution from surrounding lowactivity areas to high-activity solid organs could be neglected, absorbed dose was derived by multiplication by an appropriate dose concentration factor, resulting in absorbed doses to the kidneys ranging from 2 to $10 \mathrm{~Gy}$ per therapy cycle. This large variation could be explained by differences in tumor burden, degree of tumor uptake, and kidney function.

Bone marrow dosimetry was based on measured activity concentrations in blood samples and whole-body imaging in 2 dimensions. The total absorbed dose to the bone marrow ranged in most patients from 40 to 225 mGy per therapy cycle, with a few exceptions up to $450 \mathrm{mGy}$. As demonstrated in Figure 4, most of the absorbed dose to the bone marrow is due to blood-conveyed self-radiation. Also, cross-dose activity in the remainder of the body and tumors could in some patients contribute significantly. The blood activitymediated self-dose dominated the bone marrow dose in 167 of 200 patients.

A high tumor burden acting as an activity sink will lower the blood activity concentration and, if the bone marrow is free of tumor, the bone marrow self-dose. To some extent, this decrease will be counteracted by the higher cross-radiation that, around the large tumors, contributes to the local irradiation of the bone marrow. However, reduction of tumor burden during therapy does not necessarily lead to an increased bone marrow dose during later cycles. In fact, more than half the patients showed a decrease at later cycles (Fig. 6), sometimes by more than 50\%. There are several possible contributory explanations. In the early phase of

TABLE 1

Absorbed Doses to Solid Organs (Kidneys, Liver, and Spleen) and Maximum Tolerable Therapy Cycles

\begin{tabular}{llccccc}
\hline \multicolumn{1}{c}{ Parameter } & Organ & Number & First quartile & Median & Third quartile & Mean \\
\hline Absorbed dose (Gy) & Kidney right & 200 & 3.62 & 4.58 & 5.55 & 4.69 \\
& Kidney left & 198 & 3.38 & 4.33 & 5.29 & 4.39 \\
& Liver & 198 & 1.52 & 2.17 & 3.60 & 2.80 \\
& Spleen & 183 & 3.74 & 5.01 & 6.80 & 5.35 \\
N limit & & 200 & 3.87 & 4.53 & 5.52 & 4.79 \\
\hline
\end{tabular}




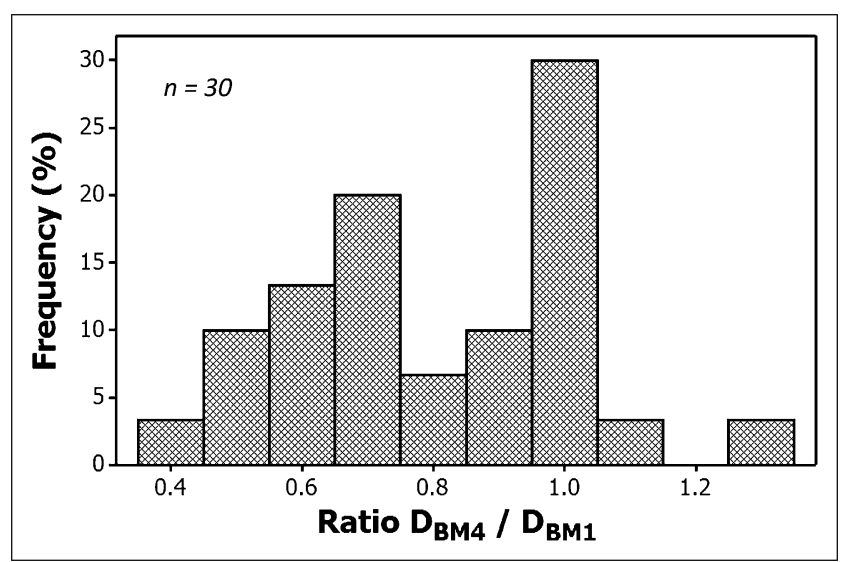

FIGURE 6. Ratios of absorbed dose of bone marrow between cycle 4 or 5 and cycle 1.

treatment, a decreased clearance of tracer can occur for various reasons: a high tumor burden stealing perfusion from healthy organs, an inflammatory reaction in the tumor, mechanical obstruction of the ureters, or excretion from the tumor of vasoactive or diarrhea-inducing hormones, leading to metabolic imbalance. In the later treatment phase, when the tumor burden has been reduced, many of these factors can improve. The faster activity clearance will then compensate for the higher initial blood activity, reducing both the kidney dose and the bone marrow dose. A case study describing this relationship in some detail was published earlier (37). This rather complex physiologic interplay indicates the need for detailed individual dosimetry and underlines the advantage offered by a fractionated therapy protocol with ${ }^{177} \mathrm{Lu}$-DOTA-octreotate. For patients with major changes in tumor burden or kidney function, we therefore advocate more frequent measurements with complete dosimetry because changes in pharmacokinetics can lead to overestimation of both kidney and bone marrow doses.

By combining these 2 dosimetry protocols, we conclude that more than $50 \%$ of the patients could receive more than the 4 cycles of $7.4 \mathrm{GBq}$ with ${ }^{177} \mathrm{Lu}$-octreotate. On the other

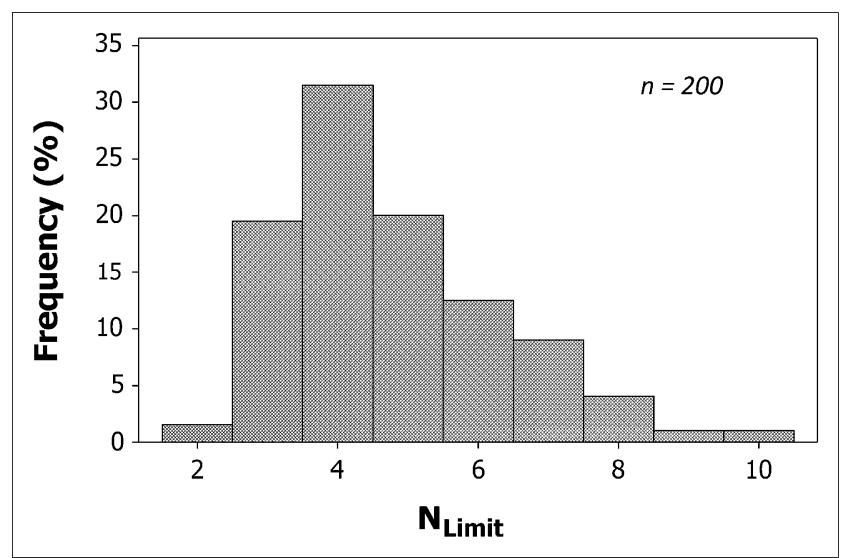

FIGURE 7. Maximum tolerable number of cycles with respect to absorbed doses to bone marrow and kidneys for 200 patients. hand, our results indicate that about $20 \%$ of the patients may tolerate fewer than the 4 cycles. To test the assumption that a considerable number of patients may be undertreated, and encouraged by the low incidence of serious late toxicity to kidneys and bone marrow as reported by the Rotterdam group, we are currently conducting a prospective study offering patients clinically responding to 4 cycles with ${ }^{177} \mathrm{Lu}-$ octreotate additional cycles based on dosimetry.

Blood sampling in the individual patient was not done consistently during all therapy cycles. Therefore, in the presented calculation of possible number of treatment cycles, some assumptions were made that will overestimate the absorbed dose to bone marrow. First, the self-dose to the bone marrow was calculated from the time-integrated blood curve assuming that the number of decays per unit mass in bone marrow and blood is the same (16). However, in most patients blood was sampled only up to $24 \mathrm{~h}$. The late phase that contributes to almost $50 \%$ of the total number of decays was measured in only 50 of 200 patients. From this dataset, a half-life of $72 \mathrm{~h}$ was derived and applied to the other patients, although $95 \%$ of the patients actually had a conceivably lower value. This was a conservative assumption because bone marrow doses tended to decrease at later therapy cycles.

The assumption that the number of decays per gram in bone marrow and blood is the same is crude and is based on merely a few measurements during the late phase (16). The possibility that the distribution factor may vary in the early blood phase, containing mainly intact ${ }^{177} \mathrm{Lu}$-octreotate, and the late phase, containing ${ }^{177} \mathrm{Lu}$-labeled catabolites, cannot be excluded. Walrand et al. (38) concluded from PET data that the bone marrow dose may have a significant contribution from catabolites. Further investigations are needed in this respect, with focus on the question of how far the absorbed doses to critical organs during therapy can be sufficiently predicted by a dosimetry model based also on PET measurements.

In a recent study of over 1,100 patients treated with ${ }^{90}$ Y-octreotide (mean of 2 cycles), about $9 \%$ of clinically significant nephrotoxicity was reported (5). This percentage is in contrast to the less than $0.5 \%$ reported for 504 patients treated with 4 cycles of ${ }^{177} \mathrm{Lu}$-octreotate (2). Besides the obvious differences in energy and half-life of the radionuclides used, there is also the variation in the number of therapy cycles. In light of this, our conclusion that more than 4 cycles of $7.4 \mathrm{GBq}$ of ${ }^{177} \mathrm{Lu}$-octreotate can be given in $50 \%$ of the patients might stimulate discussion on fractionated treatment with guidance from dosimetric data. Our finding that absorbed doses to the bone marrow tend to decrease, whereas the kidneys in general show only a modest increase of absorbed doses, may support the preference for more fractions. As long as the antitumor effect is larger than the radiation effect on healthy organs, fractionated treatment seems to be preferable. That leaves the discussion open as to the level of activity at which treatment should be started. PET studies with ${ }^{68} \mathrm{Ga}$-labeled somatostatin analogs may be helpful to estimate the total amounts of receptors expressed 
by the tumors and may thus help with finding the optimal start activity. This is a field for future investigations.

The cross-fire dose to the bone marrow was based on DF calculated in a standard adult. Application of these values to patients of various weights and anatomies renders no more than an approximation, but the error in the total absorbed dose is small since most organs contribute only to a small degree. The remainder of the body is composed of different types of tissues with genuinely different characteristics, and for that reason, dose calculations are also in this case an approximation. Commonly used software programs for absorbed dose calculations assume a homogeneous activity distribution and use a DF that includes a $\beta$-component representing $88.7 \%$ of the total. However, solid bone (compacta) that surrounds the bone marrow has a low degree of vascularization as compared with soft tissues included in the remainder of the body as well as in the adjacent red marrow and, as a consequence, a comparatively negligible activity uptake. Thus, a DF without a $\beta$-component will probably give a more correct cross-dose to bone marrow. By using a DF corrected for the $\beta$-component, we find that the crossfire contribution to bone marrow dose ranges between 10 and $150 \mathrm{mGy}$. In this context, it should be remembered that in about $10 \%$ of the patients with advanced neuroendocrine tumors, bone or bone marrow metastases occur, as was also the case in our patient material (data not shown). Bone or bone marrow metastases will cause a substantially higher absorbed dose locally to the bone marrow. Moreover, the radiation-sensitive red marrow is not homogeneously distributed, and the mean calculated dose to the bone marrow may in fact give only a vague indication of the effect to be expected. It is therefore important that the physician interprets the dosimetry report cautiously. The age of the patient and the distribution within and adjacent to the bone marrow, as well as earlier bone marrow commitments such as cytostatic treatments, will have an impact on the bone marrow response.

When applying a dose limit of $2 \mathrm{~Gy}$ for bone marrow and 23 Gy for kidneys, previous studies have estimated that the bone marrow is the dose-limiting organ in up to $70 \%$ of patients undergoing therapy with ${ }^{177} \mathrm{Lu}$-octreotate (39). The present work, however, indicates the kidney to be doselimiting in $98.5 \%$ of the patients. The difference may be due to differences in the measuring methodology and needs to be addressed further. One obvious confounder is the $\beta$-component in the applied DF.

There is an increasing insight that absorbed dose by itself may not be sufficient for clinical assessment and that the biologic impact of treatments should be considered. The biologic effective dose formalism is one such method that incorporates biologic response parameters such as sublethal damage recovery time (12). For the data presented in this work, median biologic effective dose to kidney was $4.9 \mathrm{~Gy}$ (range, 1.8-15.7 Gy) for a single treatment cycle. Since the maximum tolerated biologic effective dose is higher than the generally accepted maximum tolerated absorbed dose to the kidneys ( $\leq 45$ Gy (11) compared with 23-29 Gy, respectively), use of biologic effective dose instead of absorbed dose might affect the general conclusion of our work in terms of the number of tolerated cycles and the dose-limiting organ.

Based on the presented results, most patients (79\%) can complete 4 cycles of treatment with $7.4 \mathrm{GBq}$ of ${ }^{177} \mathrm{Lu}-$ octreotate. If the maximum tolerable dose to the kidney is increased to $29 \mathrm{~Gy}$, as has been suggested (24), nearly all patients $(97 \%)$ can complete 4 cycles. Even with the 23-Gy limit, about half of patients can undergo 5 treatment cycles or more. However, the variations in the maximum tolerable number of cycles per patient confirm that individual calculations of the absorbed dose are required to ensure optimal treatment.

\section{CONCLUSION}

On the basis of whole-body imaging in combination with blood and urine activity curves, a method for determination of the absorbed dose to the bone marrow was developed. The dose-limiting organ was the kidney in 197 of 200 patients. Twenty-eight of 30 patients showed a decreased or stable absorbed dose to the bone marrow at later cycles. In $50 \%$ of the patients more than 4 cycles, and in $20 \%$ fewer than 4 cycles, of $7.4 \mathrm{GBq}{ }^{177} \mathrm{Lu}$-octreotate could be administered. Individualized absorbed dose calculations are essential to optimizing therapy.

\section{DISCLOSURE}

The costs of publication of this article were defrayed in part by the payment of page charges. Therefore, and solely to indicate this fact, this article is hereby marked "advertisement" in accordance with 18 USC section 1734 . No potential conflict of interest relevant to this article was reported.

\section{ACKNOWLEDGMENTS}

We acknowledge with gratitude Prof. Eric Krenning, Erasmus Medical Centre, Rotterdam, for the generous supply of the peptide, and the staff at the Department of Medical Physics, Nuclear Medicine, Pharmacy, and at the Department of Endocrine Oncology (78D) for their considerable assistance.

\section{REFERENCES}

1. Kwekkeboom DJ, Teunissen JJ, Bakker WH, et al. Radiolabeled somatostatin analog $\left[{ }^{177} \mathrm{Lu}\right.$-DOTA0,Tyr3]octreotate in patients with endocrine gastroenteropancreatic tumors. J Clin Oncol. 2005;23:2754-2762.

2. Kwekkeboom DJ, de Herder WW, Kam BL, et al. Treatment with the radiolabeled somatostatin analog [177 Lu-DOTA 0,Tyr3]octreotate: toxicity, efficacy, and survival. J Clin Oncol. 2008;26:2124-2130.

3. Bodei L, Cremonesi M, Grana CM, et al. Peptide receptor radionuclide therapy with ${ }^{177}$ Lu-DOTATATE: the IEO phase I-II study. Eur J Nucl Med Mol Imaging. 2011;38:2125-2135.

4. Gabriel M, Andergassen U, Putzer D, et al. Individualized peptide-relatedradionuclide-therapy concept using different radiolabelled somatostatin analogs in advanced cancer patients. Q J Nucl Med Mol Imaging. 2010;54:92-99.

5. Imhof A, Brunner P, Marincek N, et al. Response, survival, and long-term toxicity after therapy with the radiolabeled somatostatin analogue [ $\left.{ }^{90} \mathrm{Y}-\mathrm{DOTA}\right]-$ TOC in metastasized neuroendocrine cancers. J Clin Oncol. 2011;29:2416-2423. 
6. Williams LE, DeNardo GL, Meredith RF. Targeted radionuclide therapy. Med Phys. 2008;35:3062-3068.

7. Moll S, Nickeleit V, Mueller-Brand J, Brunner FP, Maecke HR, Mihatsch MJ. A new cause of renal thrombotic microangiopathy: yttrium 90-DOTATOC internal radiotherapy. Am J Kidney Dis. 2001;37:847-851.

8. Valkema R, Pauwels SA, Kvols LK, et al. Long-term follow-up of renal function

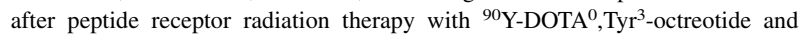
${ }^{177}$ Lu-DOTA $^{0}$, Tyr $^{3}$-octreotate. J Nucl Med. 2005;46(suppl 1):83S-91S.

9. Bodei L, Cremonesi M, Grana C, et al. Receptor radionuclide therapy with ${ }^{90} \mathrm{Y}-$ [DOTA]0-Tyr3-octreotide ( ${ }^{90}$ Y-DOTATOC) in neuroendocrine tumours. Eur J Nucl Med Mol Imaging. 2004;31:1038-1046.

10. Bodei L, Cremonesi M, Ferrari M, et al. Long-term evaluation of renal toxicity after peptide receptor radionuclide therapy with ${ }^{90} \mathrm{Y}$-DOTATOC and ${ }^{177} \mathrm{Lu}$-DOTATATE: the role of associated risk factors. Eur J Nucl Med Mol Imaging. 2008;35: 1847-1856.

11. Barone R, Borson-Chazot F, Valkema R, et al. Patient-specific dosimetry in predicting renal toxicity with ${ }^{90}$ Y-DOTATOC: relevance of kidney volume and dose rate in finding a dose-effect relationship. J Nucl Med. 2005;46(suppl 1): 99S-106S.

12. Siegel JA, Stabin MG, Sharkey RM. Renal dosimetry in peptide radionuclide receptor therapy. Cancer Biother Radiopharm. 2010;25:581-588.

13. Lassmann M, Chiesa C, Flux G, Bardies M. EANM Dosimetry Committee guidance document: good practice of clinical dosimetry reporting. Eur J Nucl Med Mol Imaging. 2011;38:192-200.

14. Hindorf C, Glatting G, Chiesa C, Linden O, Flux G. EANM Dosimetry Committee guidelines for bone marrow and whole-body dosimetry. Eur J Nucl Med Mol Imaging. 2010;37:1238-1250.

15. Ljungberg M, Sjogreen-Gleisner K. The accuracy of absorbed dose estimates in tumours determined by quantitative SPECT: a Monte Carlo study. Acta Oncol. 2011;50:981-989.

16. Forrer F, Krenning EP, Kooij PP, et al. Bone marrow dosimetry in peptide receptor radionuclide therapy with $\left[{ }^{177} \mathrm{Lu}-\mathrm{DOTA}^{0}, \mathrm{Tyr}^{3}\right]$ octreotate. Eur J Nucl Med Mol Imaging. 2009;36:1138-1146.

17. Coleman CN, Blakely WF, Fike JR, et al. Molecular and cellular biology of moderate-dose (1-10 Gy) radiation and potential mechanisms of radiation protection: report of a workshop at Bethesda, Maryland, December 17-18, 2001. Radiat Res. 2003;159:812-834.

18. Wehrmann C, Senftleben S, Zachert C, Muller D, Baum RP. Results of individual patient dosimetry in peptide receptor radionuclide therapy with ${ }^{177} \mathrm{Lu}$ DOTATATE and ${ }^{177}$ Lu DOTA-NOC. Cancer Biother Radiopharm. 2007;22:406-416.

19. Garkavij M, Nickel M, Sjogreen-Gleisner K, et al. ${ }^{177}$ Lu-[DOTA0,Tyr3] octreotate therapy in patients with disseminated neuroendocrine tumors: analysis of dosimetry with impact on future therapeutic strategy. Cancer. 2010;116:1084-1092.

20. Sandström M, Garske U, Granberg D, Sundin A, Lundqvist H. Individualized dosimetry in patients undergoing therapy with ${ }^{177} \mathrm{Lu}-\mathrm{DOTA}-\mathrm{D}-\mathrm{Phe}$ (1)-Tyr (3)-octreotate. Eur J Nucl Med Mol Imaging. 2010;37:212-225.

21. Swärd C, Bernhardt $P$, Ahlman H, et al. [ ${ }^{177}$ Lu-DOTA 0-Tyr 3]-octreotate treatment in patients with disseminated gastroenteropancreatic neuroendocrine tumors: the value of measuring absorbed dose to the kidney. World J Surg. 2010;34:1368-1372.

22. Claringbold PG, Brayshaw PA, Price RA, Turner JH. Phase II study of radiopeptide ${ }^{177} \mathrm{Lu}$-octreotate and capecitabine therapy of progressive disseminated neuroendocrine tumours. Eur J Nucl Med Mol Imaging. 2011;38:302-311.
23. Emami B, Lyman J, Brown A, et al. Tolerance of normal tissue to therapeutic irradiation. Int J Radiat Oncol Biol Phys. 1991;21:109-122.

24. Konijnenberg M, Melis M, Valkema R, Krenning E, de Jong M. Radiation dose distribution in human kidneys by octreotides in peptide receptor radionuclide therapy. J Nucl Med. 2007;48:134-142.

25. Garske U, Sandström M, Johansson S, et al. Minor changes in effective half-life during fractionated ${ }^{177} \mathrm{Lu}$-octreotate therapy. Acta Oncol. 2012;51:86-96.

26. Stabin MG, Siegel JA, Sparks RB, Eckerman KF, Breitz HB. Contribution to red marrow absorbed dose from total body activity: a correction to the MIRD method. J Nucl Med. 2001;42:492-498.

27. Home page. RADAR Web site. http://www.doseinfo-radar.com/. Accessed November 16, 2012.

28. Lassmann M, Strigari L, Bardies M. Dosimetry is alive and well. Cancer Biother Radiopharm. 2010;25:593-595.

29. Wessels BW, Dale RG, Cremonesi M, et al. Renal dosimetry. Cancer Biother Radiopharm. 2010;25:597-599.

30. Bodei L, Cremonesi M, Paganelli G. Something more than estimating renal dosimetry. Cancer Biother Radiopharm. 2010;25:767-768.

31. Siegel JA, Stabin MG, Sharkey RM. Renal dosimetry: ready for biological equivalent dose? Response to medical internal radiation dose and European association of nuclear medicine committee letters. Cancer Biother Radiopharm. 2010;25:763-765.

32. Cremonesi M, Botta F, Di Dia A, et al. Dosimetry for treatment with radiolabelled somatostatin analogues: a review. Q J Nucl Med Mol Imaging. 2010; $54: 37-51$

33. Wessels BW, Konijnenberg MW, Dale RG, et al. MIRD pamphlet no. 20: the effect of model assumptions on kidney dosimetry and response-implications for radionuclide therapy. J Nucl Med. 2008;49:1884-1899.

34. Rolleman EJ, Melis M, Valkema R, Boerman OC, Krenning EP, de Jong M. Kidney protection during peptide receptor radionuclide therapy with somatostatin analogues. Eur J Nucl Med Mol Imaging. 2010;37:1018-1031.

35. Khan S, Krenning EP, van Essen M, Kam BL, Teunissen JJ, Kwekkeboom DJ. Quality of life in 265 patients with gastroenteropancreatic or bronchial neuroendocrine tumors treated with $\left[{ }^{177} \mathrm{Lu}-\mathrm{DOTA}\right.$, Tyr3]octreotate. J Nucl Med. 2011;52:1361-1368.

36. De Jong M, Valkema R, Jamar F, et al. Somatostatin receptor-targeted radionuclide therapy of tumors: preclinical and clinical findings. Semin Nucl Med. 2002; 32:133-140.

37. Garske U, Sandstrom M, Johansson S, et al. Lessons to learn on tumour response: imaging during therapy with ${ }^{177} \mathrm{Lu}$-DOTA-octreotate: a case report on a patient with a large volume of poorly differentiated neuroendocrine carcinoma. Theranostics. 2012;2:459-471.

38. Walrand S, Barone R, Pauwels S, Jamar F. Experimental facts supporting a red marrow uptake due to radiometal transchelation in ${ }^{90}$ Y-DOTATOC therapy and relationship to the decrease of platelet counts. Eur J Nucl Med Mol Imaging. 2011;38:1270-1280.

39. Esser JP, Krenning EP, Teunissen JJ, et al. Comparison of $\left[{ }^{177} \operatorname{Lu}-\mathrm{DOTA}(0), \mathrm{Tyr}\right.$ (3)]octreotate and $\left[{ }^{177} \mathrm{Lu}-\operatorname{DOTA}(0), \operatorname{Tyr}(3)\right]$ octreotide: which peptide is preferable for PRRT? Eur J Nucl Med Mol Imaging. 2006;33:1346-1351. 